\title{
LOS RECURSOS RESIDENCIALES PARA LA TRANSICIÓN HACIA LA VIDA ADULTA DE LOS JÓVENES TUTELADOS EN CATALUÑA
}

\author{
RESIDENTIAL RESOURCES FOR THE TRANSITION TOWARDS ADULT \\ LIFE FOR FOSTERED YOUTHS IN CATALONIA
OS RECURSOS RESIDENCIAIS PARA A TRANSIÇÃO PARA A VIDA ADULTA DOS JOVENS TUTELADOS EM CATALUÑA

\author{
Antonio COMASÒLIVAS MOYA*, Josefina SALA-ROCA* \\ \& Teresa Eulàlia MARZO ARPÓN** \\ *Universidad Autónoma de Barcelona (UAB), ${ }^{* *}$ Universitat Ramon Llull
}

PALABRAS CLAVE: jóvenes tutelados extutelados ayuda a la emancipación autonomía transición a la vida adulta
RESUMEN: Los jóvenes tutelados y extutelados en Cataluña pueden acceder al programa de vivienda de apoyo a la emancipación desde los 16 a los 21 años. El objetivo de este estudio es describir los recursos residenciales de transición a la vida adulta para los jóvenes extutelados en Cataluña, así como el perfil de los jóvenes, sus educadores y la labor que en éstos se realiza. Para ello se establecieron 5 metacategorías: Datos identificativos de la entidad y sus recursos, características de los jóvenes residentes, aspectos educativos y de apoyo al joven, acceso y permanencia en el recurso y salida del recurso. Se entrevistó a 26 profesionales de las entidades que gestionan estos recursos residenciales mediante entrevistas semiestructuradas que fueron transcritas y codificadas para realizar posteriormente un análisis centrado en las categorías propuestas en el diseño. Los profesionales apuntaron que los jóvenes que permanecen en el programa son aquellos que tienen un proyecto claro de emancipación y aceptan las orientaciones educativas y las reglas el recurso residencial. También apuntaron a que tener un proyecto educativo y tener un proceso formativo en curso, aumentaba el tiempo de permanencia en el programa y que la falta de oportunidades laborales disuadía a los jóvenes a abandonar el programa, ya que las prestaciones económicas que reciben no les aporta la suficiente estabilidad financiera. En cuanto a las razones para abandonar el programa, mencionaron la estabilidad económica y laboral. Debido a la situación social y económica y la falta de oportunidades de trabajo, la mayoría de los jóvenes abandonan el programa sin haber tenido la oportunidad de demostrar su valía.

CONTACTAR CON LOS AUTORES: Josefina Sala-Roca. Correo electrónico: Fina.Sala@uab.cat Dirección postal: Facultat de Ciències de l'Educació, despacho G6-171 Universitat Autònoma de Barcelona. 08193 Cerdanyola del Vallès. 


\begin{tabular}{|c|c|}
\hline $\begin{array}{l}\text { KEY WORDS: } \\
\text { foster care } \\
\text { care leavers } \\
\text { emancipation } \\
\text { programs } \\
\text { autonomy } \\
\text { transition to } \\
\text { adulthood }\end{array}$ & $\begin{array}{l}\text { ABSTRACT: In Catalonia, Youths in care and Care leavers can apply for a housing program } \\
\text { from } 16 \text { up to } 21 \text { to support their emancipation process. The aim of this study is to describe the } \\
\text { housing program for transitioning to adult life for former foster youths in Catalonia, as well as } \\
\text { the profile of young people, their educators and the work carried out in them. To this end, } 5 \\
\text { methacategories were established: Identification data of the entity and its housing program, } \\
\text { characteristics of the young residents, educational and support aspects to the youth, access } \\
\text { and permanence in the housing program and leaving of the housing program. A semi-struc- } \\
\text { tured interview was used to interview } 26 \text { professionals. Professionals pointed out that the } \\
\text { youth who stay longer are the ones who have a clear emancipation project and accept the } \\
\text { educative supervision and program rules. The professionals explained that having an educa- } \\
\text { tive project and being enrolled in a training process increase the stay, because the financial } \\
\text { subsidises they receive don't provide enough financial stability. Professionals pointed out that } \\
\text { reasons for leaving the program were the lack of labour opportunities because subsidises } \\
\text { they receive don't brings them enough financial stability. The reasons to left the program were } \\
\text { the economic and labour stability. Due to the social and economic situation and the lack of } \\
\text { job opportunities, most young people leave the program without having had the opportunity } \\
\text { to prove themselves. }\end{array}$ \\
\hline $\begin{array}{l}\text { PALAVRAS-CHAVE: } \\
\text { jovens tutelados } \\
\text { extutelados } \\
\text { ajuda à emancipação } \\
\text { autonomia } \\
\text { transição à vida } \\
\text { adulta }\end{array}$ & $\begin{array}{l}\text { RESUMO: Os jovens tutelados e extutelados em Catalunha podem aceder ao programa resi- } \\
\text { dencial de apoio à emancipação dos os } 16 \text { aos } 21 \text { anos. } \\
\text { O objectivo deste estudo é descrever os recursos residenciais de transição à vida adulta para } \\
\text { os jovens extutelados em Catalunha, bem como definir o perfil dos jovens, dos educadores e } \\
\text { o trabalho que estes realizam. } \\
\text { Para isso estabeleceram-se } 5 \text { meta categorias: Dados identificativos da entidade e seus recur- } \\
\text { sos, características dos jovens residentes, aspetos educativos e de apoio ao jovem, acesso e } \\
\text { permanência na casa de acolhimento e respetiva saída. } \\
\text { Foram entrevistados } 26 \text { profissionais das entidades que gerem estes recursos residenciais } \\
\text { mediante entrevistas semiestruturadas que foram transcritas e codificadas para realizar pos- } \\
\text { teriormente uma análise centrada nas categorias propostas no desenho. } \\
\text { Os profissionais apontaram que os jovens que permanecem no programa são aqueles que } \\
\text { têm um projeto claro de emancipação e aceitam as orientações educativas e as regras da } \\
\text { casa de acolhimento. } \\
\text { Também apontaram que ter um projeto educativo e ter um processo formativo em curso, } \\
\text { aumentava o tempo de permanência no programa, e que, a falta de oportunidades de tra- } \\
\text { balho dissuadia os jovens a abandonarem o programa, já que as prestações económicas que } \\
\text { recebem não lhes dariam a estabilidade financeira desejada. } \\
\text { Quanto às razões para abandonar o programa, mencionaram a estabilidade económica e o } \\
\text { trabalho. Devido à situação social e económica e à falta de oportunidades de trabalho, a maio- } \\
\text { ria dos jovens abandonam o programa sem ter tido a oportunidade de demonstrar sua valia. }\end{array}$ \\
\hline
\end{tabular}

\section{Introducción}

En el año 2014, 42.867 niños y jóvenes españoles estaban bajo la tutela de las administraciones públicas (Observatorio de la infancia, 2016). Esta medida de protección finaliza en cuanto el joven llega a su mayoría de edad. Melendro (2010 y 2015) señala el concepto de "jóvenes adultos", utilizado en diversas investigaciones de los años dos mil para hacer referencia a aquellos jóvenes que no han transitado de forma clara a la vida adulta autónoma y establece diferentes factores que inciden en la independencia: laboral, social, familiar y económicos, los cuales van logrando de forma no lineal y cada vez de forma más tardía. La transición a la vida adulta es especialmente difícil para los jóvenes tutelados, pero aún lo es más en un país con altas tasas de paro juvenil y dificultades de acceso a la vivienda como es el caso de España. Es por ello que los jóvenes tutelados se enfrentan a una situación de transición más breve, acelerada y comprimida hacia la vida adulta que sus pares (Lopez, Santos, Bravo y Fernández del Valle, 2013). Esta transición además debe realizarse con una red de apoyo pobre (Bravo \& Fernández del Valle, 2001; Bravo \& Fernández del Valle, 2003; Martín, 2011; Martín, García \& Siverio, 2012; Martín, Muñoz, Rodríguez \& Pérez, 2008; Sala, ViIlalba, Jariot \& Arnau, 2012) muy necesaria para la transición exitosa a la vida adulta (Montserrat, Casas, González, Malo, Araujo \& Navarro, 2010). Frecuentemente los jóvenes se orientan a su familia sin que esta cuente con la capacidad para apoyar al joven (París, Hernando \& Martínez, 2017).

Pero las dificultades no se constriñen solo al contexto socioeconómico hostil y a la edad temprana en que los jóvenes tutelados por la Administración deben independizarse de su "padre corporativo" (Courtney, 2009); sino que la gran mayoría deben realizar este difícil paso con unos niveles educativos bajos, baja cualificación laboral y menor red social de apoyo. De hecho, la 
mayoría de los jóvenes tutelados tienen problemas de subempleo, inestabilidad laboral, y pobreza (Courtney \& Dworsky, 2006, Courtney et al, 2005; Courtney et al, 1998, 2001, Jariot, Sala \& Arnau, 2015; entre otros). Un meta análisis de 32 estudios señala disponer de un hogar estable y la educación como los factores más determinantes (Gypen, Vanderfaeillie, De Maeyer, Belenger, \& Van Holen, 2017).

En un país con una alta tasa de paro juvenil, el nivel educativo es el primer filtro que se utiliza para seleccionar los posibles candidatos a un puesto de trabajo. Diversos estudios internacionales señalan que los jóvenes tutelados tienen niveles educativos muy inferiores a sus pares (Courtney, 2009; Courtney et al, 2001; Courtney \& Dworsky, 2006; Festinger 1983; Pecora et al, 2006), así como porcentajes muy inferiores de obtención del graduado en la ESO respecto a la población general (Montserrat, Casas, Malo \& Bertran, 2011; Montserrat, Casas, Malo, 2013; Montserrat, Casas \& Bertran, 2013; Montserrat, Casas y Baena, 2015; Sala, Villaba, Jariot \& Rodríguez, 2009).

Son varios los factores que inciden en esta baja tasa de graduación: historias previas de desescolarización en la familia de origen, altos índices de discapacidad y trastornos psíquicos, bajo apoyo educativo, malestar emocional que experimentan, cambios continuos de centros escolares, y que se priorizan los itinerarios formativos no reglados debido las bajas expectativas formativas que tienen los profesionales que atienden a los jóvenes y a la situación de tener que subsistir por sí mismos una vez cumplidos los 18 años (Casas, Montserrat \& Malo, 2010; Courtney, 2009; Fernández del VaIle, Sinero \& Bravo, 2011; IARS, 2009; Sala, Villalba, Jariot \& Arnau, 2012).

De hecho, Courtney, Hook y Lee (2010) observaron que los jóvenes extutelados con un nivel educativo más elevado, tienen mayores probabilidades de encontrar empleo, de tener una vivienda estable, de evitar problemas con la justicia y una menor probabilidad de maternidad/paternidad adolescente. Abandonar el sistema de protección genera, normalmente, salarios más bajos y mayores niveles de paro y en consecuencia mayores situaciones de pobreza (Courtney \& Dworsky, 2006). Hook y Courtney (2011) demostraron que permanecer más tiempo en el sistema de protección puede actuar como elemento protector ante estas situaciones. En esta misma línea, los resultados del metaanálisis realizado por Heerde, Hemphill, y Scholes-Balog, (2016) subrayan los beneficios de los programas de transición en vivienda, empleo y educación.
Es por ello que de forma pionera en España, en Cataluña se empezaron a configurar en 1994 servicios de apoyo a la transición que se han ido desarrollando, y actualmente están bajo la dirección del ASJTET (Área de Apoyo a los Jóvenes Tutelados y Extutelados) y recogidos en la Ley de Derechos y Oportunidades de la Infancia 14/2010. El ASJTET ofrece diferentes servicios (supervisión socioeducativa, asesoramiento legal, asesoramiento laboral, vivienda y apoyo económico) orientados a jóvenes tutelados y extutelados mayores de 16 años que no tengan posibilidad de volver al núcleo familiar y que presenten un riesgo alto de exclusión al cumplir los 18 años. Durante el año 2014, 537 jóvenes se beneficiaron del programa de vivienda (Generalitat de Catalunya, 2015). La mayoría de estas viviendas son pequeñas unidades supervisadas por una gran diversidad de entidades (fundaciones, cooperativas o asociaciones) conveniadas con el ASJTET. Gran parte de estas entidades están adheridas a la Federación de Entidades con Proyectos y Pisos Asistidos (FEPA), una organización de ámbito estatal. Es por ello, que los programas de apoyo a la transición a la vida adulta para jóvenes tutelados de Cataluña son un referente a considerar para la implantación de estos servicios en otras comunidades autónomas.

El objetivo de este estudio es describir los recursos residenciales de transición a la vida adulta para los jóvenes extutelados en Cataluña, así como el perfil de los jóvenes, sus educadores y la labor que en éstos se realiza. Para ello se establecieron 5 metacategorías: Datos identificativos de la entidad y sus recursos, características de los jóvenes residentes, aspectos educativos y de apoyo al joven, acceso y permanencia en el recurso y salida del recurso.

\section{Metodología}

El estudio que se ha realizado es un estudio descriptivo que persigue recabar datos que nos permitan conocer las características de los programas de vivienda para jóvenes extutelados y los perfiles de los jóvenes. La metodología es predominantemente cualitativa, si bien también se han recogido algunos datos cuantitativos. El diseño utilizado es el transaccional descriptivo. Para recabar la información se diseñó una entrevista semiestructurada en la que se preguntaba a las entidades acerca del número de recursos, plazas, perfil de jóvenes atendidos, perfil de educadores, finalidades y estrategias de intervención, tiempo de permanencia en el recurso y las causas de abandono. 
La entrevista fue validada por 4 expertos en este ámbito de investigación. Así mismo, se solicitó al ASJTET permiso para el desarrollo del estudio. El diseño del estudio fue aprobado por la Comisión Ética de Experimentación Animal y Humana de la Universidad Autónoma de Barcelona (CEEAH) con el informe número 2272.

Se entrevistaron a profesionales de 25 de las 29 entidades que gestionan 59 recursos residenciales para jóvenes extutelados en Cataluña. Se estableció como criterio principal que éstas tuvieran, por lo menos, un recurso residencial exclusivamente para jóvenes extutelados y como criterio para seleccionar a la persona a entrevistar se solicitó que ésta fuera un directivo o un profesional con cargos de gestión dentro de los recursos residenciales para que existiera una representatividad de carácter conceptual que pudiera dar respuesta a todos los ítems planteados. Se contactó telefónicamente con dichas entidades para solicitarles una entrevista con un profesional con cargo de responsabilidad en la entidad y se acordó un día para realizar la entrevista. Previamente al desarrollo de ésta se informó a la persona entrevistada del contenido de la misma, de los objetivos del estudio, y se solicitó su participación voluntaria. También se entrevistó a un referente del ASJTET para conocer los criterios de acceso y derivación de los casos. Las entrevistas fueron grabadas para su posterior transcripción.

Los datos recogidos fueron analizados mediante matrices descriptivas. El método de análisis fue el análisis de contenido de tipo categorial. El proceso de categorización fue mixto deductivo-inductivo.

\section{Resultados}

\subsection{Recursos Residenciales de transición, requisitos de acceso y permanencia}

Una de las principales características del programa de vivienda es la fragmentación y los formatos pequeños. Los 59 recursos residenciales analizados pertenecen a 25 entidades que tienen convenidas sus plazas con la Administración mayoritariamente (89\%). Solo 6 recursos tienen una infraestructura de propiedad pública pero su gestión está delegada a una entidad privada. Así, encontramos entidades que gestionan un solo recurso $(28 \%)$, otras gestionan dos o tres recursos $(60 \%)$ y unas pocas gestionan 4 o más recursos (12\%).

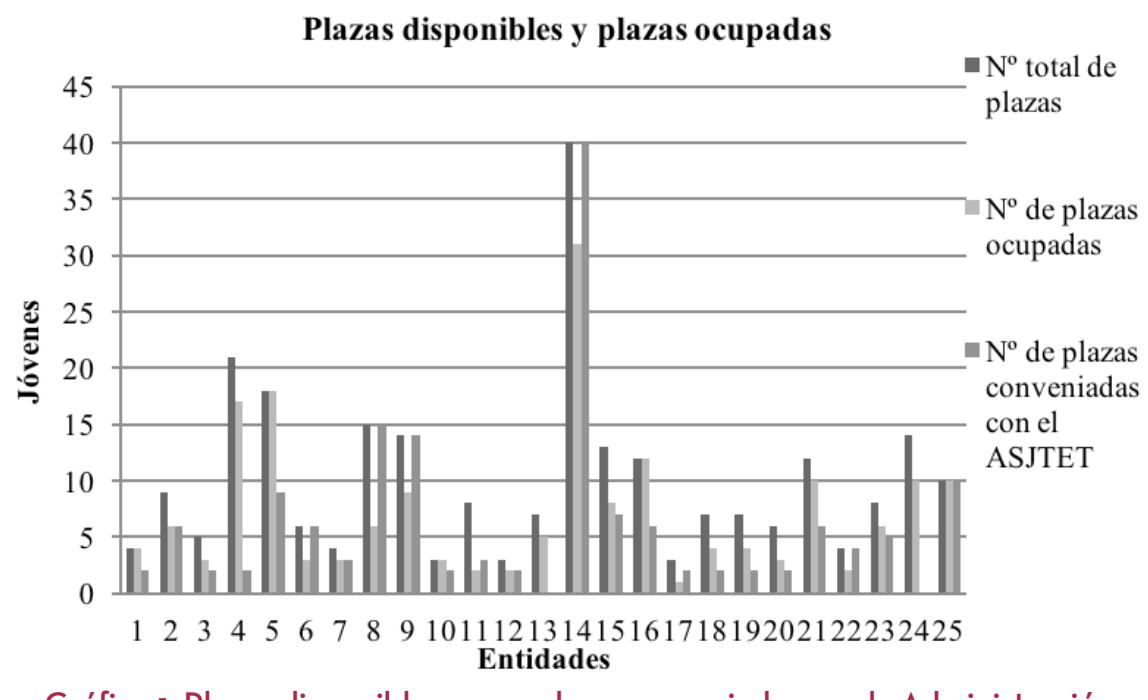

Gráfico 1. Plazas disponibles, ocupadas y conveniadas con la Administración

En total estas entidades ofertan 267 plazas, de las cuales estaban ocupadas en el momento de la entrevista 196 (73\% de las disponibles). Los recursos disponen de entre 2 y 7 plazas. Predominan las viviendas de 4-5 plazas (49\%), seguidas de las de 2-3 plazas (37\%) y las viviendas de 6-8 plazas o de más de 8 plazas son poco habituales (7\% en ambos casos). Estos formatos pequeños persiguen crear entornos lo más normalizados posibles en los que los jóvenes deben responsabilizarse de su vivienda y convivir con otros jóvenes:
Aquí lo único que tengo, que no se asemeja a un piso es el cuadrante de limpieza, que cuando son 3 hay tres; y cuando son dos se lo combinan entre las dos. Pero a partir de aquí, todo lo demás funciona como un piso normal de estudiantes [...] También quiero que ellas sepan que están en su casa y que yo estoy en un espacio que no las quiero molestar. (Entidad 12)

La supervisión educativa es intrínseca a todos los recursos, siendo mayoritariamente visitas puntuales a la vivienda, pero disponibilidad telefónica 
diaria $(88 \%)$ y solo en unos pocos $(12 \%)$, los dirigidos a jóvenes menos autónomos, ofrecen atención educativa las 24 horas del día.

El joven que desea acceder a estos recursos tiene que solicitarlo al ASJTET antes de su mayoría de edad, y debe elaborar un proyecto educativo y demostrar la adquisición de unas mínimas competencias de autonomía. Los técnicos de ASJTET valoran la candidatura y la proponen al recurso residencial. Generalmente es aceptada por que los técnicos han demostrado, en su trayectoria, el acierto en la selección de jóvenes, aunque existe la posibilidad de rechazarla. Finalmente se propone el recurso al interesado

[...] Entonces, ya hay una preselección [...] se les ofrece generalmente solo a los chavales que alguien o los educadores de los CRAE o los técnicos del Àrea de Suport creen que puede funcionar, o sea, que no hay [...] plazas para todos los que salen de los CRAE. Entonces, hay una población que ya es un poco elegida o elitista o que se ve que tienen posibilidades de funcionar. (Entidad 4)

Otro de los requisitos que suelen pedir los centros, en el caso de jóvenes inmigrantes, es disponer del permiso de residencia o en proceso de trámite, ya que es un requisito necesario para acceder a cursos u ofertas de trabajo y si no se dispone de él, dificulta el trabajo que los educadores pueden realizar con estos jóvenes.

[...] en general todos mis chicos que entran en pisos tienen residencia, si no intento no cogerlos porque el proceso es muy largo. Yo siempre les digo que más vale que estén en el centro, ya que hay más experiencia educativa, pueden hacer talleres y cosas. $\mathrm{Si}$ no tiene permiso de residencia no pueden realizar ningún curso; se aburren. Claro yo aprieto por aquí: "¿No tiene residencia? bueno, pues lo quiero cuando la tenga". (Entidad 8)

Por último, otro de los criterios frecuentes de los recursos para aceptar a un joven es que este no presente cuadros psiquiátricos graves o problemas notorios con el consumo de drogas por falta de personal especializado que pueda ofrecer un acompañamiento educativo de calidad.

Una vez en el recurso, se requiere al joven el compromiso y aprovechamiento de éste, la aceptación del trabajo educativo y cumplir con la normativa básica necesaria para la convivencia. También se pone de manifiesto la flexibilidad de estos criterios en función de la situación y circunstancias del joven como forma de trabajo individualizado.
Si ponemos el filtro muy exagerado no entra nadie. Mira, una joven desapareció dos semanas durante el verano y eso era motivo suficiente para que perdiera la plaza, pero valoré que no era lo correcto y es la joven que ahora está estudiando, tiene trabajo y está viviendo sola en un piso alquilado. Hay que saber dar oportunidades. Son unos criterios flexibles y muy individuales. No aplicamos la norma como norma, la aplicamos a la persona y yo creo que eso es básico. (Entidad 16).

\subsection{Los profesionales}

Las entidades entrevistadas tenían un total de 61 trabajadores en plantilla con funciones educativas, lo que equivale a una media de 1 educador por recurso y una ratio promedio de un educador por cada 4.5 jóvenes. Más de la mitad de los educadores se encuentran en la franja de edad de los 30 a 40 años $(50,82 \%)$ y solamente un $4,9 \%$ supera los 50 años. Esto refleja la intención de las entidades de disponer de un personal joven, pero con la suficiente edad como para distanciarse de los jóvenes usuarios. Predomina con un 69\% el género femenino, respecto al masculino con el $31 \%$ restante. En cuanto a la formación, más de la mitad de los profesionales (64\%) eran Educadores Sociales, una cuarta parte tenía otras carreras universitarias $(28 \%)$ y algunos han realizado el $\mathrm{Ci}$ clo Formativo de Grado Superior de Integración Social o de Animación Sociocultural (8\%).

\subsection{Finalidades y estrategias de intervención en los recursos residenciales}

Todos los entrevistados coinciden en que la finalidad de los recursos residenciales es el acompañamiento hacia la autonomía del joven, por ello, uno de los principales ámbitos de trabajo en todas las entidades es la inserción laboral. Debido a la coyuntura económica, las entidades plantean la inserción laboral como algo poco asequible y centran sus esfuerzos en la formación y en que los jóvenes dispongan de recursos suficientes para poderse enfrentar a su situación en el momento de la salida; hecho que se considera desproporcionado para la edad de estos jóvenes.

[...] no es que los chicos salgan de aquí con trabajo y con piso... no, porque no se pueden llevar a cabo esos objetivos, son muy difíciles. Los objetivos son que los chicos tengan las herramientas necesarias para vivir solos, que sean capaces para enfrentarse a una entrevista de trabajo, que sean capaces en cierta forma de competir por un puesto de trabajo y sepan convivir en un espacio donde no hay educadores y puedan vivir solos. (Entidad 9) 
Otro ámbito de acompañamiento es el apoyo emocional. Las entidades proporcionan una figura adulta de referencia que les ayude y les aporte seguridad ante las dudas, inquietudes o compartir éxitos, cambios o propuestas de futuro. Los educadores resaltan una mayor necesidad de este acompañamiento con las chicas, para empoderarlas y minimizar la dependencia a la pareja para construir su proyecto personal.

Que sean capaces de vivir solas, sacarse las castañas del fuego, tener su red de amistades y que no necesariamente la amistad pase por la relación sexual o por la relación de pareja. [...] Creando una red de amigos que han sido significativos y que la cuiden pero que no tengan que depender de alguien, tanto afectivamente como económicamente. En la mujer creo que sobretodo es la autonomía de no ser más por estar con un hombre o con una mujer. (Entidad 18)

La gestión económica es un ámbito específico que todas las entidades trabajan. El joven desarrolla un plan de gastos conjuntamente con su tutor, en el que se incluye el dinero necesario para pagar la comida, gastos cotidianos y el copago del servicio que no cubre todos los gastos del piso, pero sirve para trabajar educativamente la gestión del dinero y acercar al joven a los costes reales de la vivienda. Este copago varía entre entidades (desde $45 €$ a $250 €$ ) y también en función de la economía del joven. En ningún caso, el hecho de no recibir ningún ingreso y no poder hacer frente a los gastos del copago y la manutención es motivo del cese del joven en el recurso. En caso de no poder mantenerse, las entidades lo ayudan con la manutención y cubriendo sus gastos.

Los objetivos de intervención educativa de cada joven se plasman en un plan de trabajo individualizado y su progreso se informa trimestralmente a la Administración buscando la implicación del joven en este proceso. Esta implicación es percibida de forma diferente en función del centro: algunas entidades insisten en que el proyecto debe surgir del joven y no ser impuesto, y otras esperan que los jóvenes sigan las indicaciones de los profesionales.

El itinerario lo tiene que marcar él, pero muchas veces les falta las herramientas. Entonces la faena que se puede hacer desde los pisos es acompañar este proceso, evidentemente no imponerlo, lo tiene que hacer él y creérselo él. Como educador, le tienes que poder dar esas herramientas e instrumentos para que él también pueda decidir. (Entidad 14)

Pues que, si yo le digo que vaya a algún sitio, lo haga y si le digo que haga un curso de castellano que vaya; que no tenga que estar yo pendiente de si se tiene que levantar a una hora u otra. (Entidad 8)

Las entidades también recurren a recursos externos, para cubrir las necesidades del joven, como servicios de atención al inmigrante, servicios psicológicos, servicios formativo-laborales municipales, pero también las necesidades de los equipos, como asesoramiento de equipos educativos, entre otros.

\subsection{Perfil de los jóvenes extutelados en recursos residenciales de transición a la vida adulta}

El número de jóvenes que utilizaba los recursos residenciales en el momento de la entrevista era de 196; siendo la proporción de chicos mayor que el de chicas (58.2\% vs. $41.8 \%$ ). Pocos recursos (11.9\%) son mixtos; en parte para evitar la convivencia entre jóvenes de distinto sexo, siendo una etapa en la que las relaciones afectivas impactan en el desarrollo del proyecto personal, en un espacio sin presencia continua de un referente adulto.

Claro, en un piso donde no hay un educador las 24 horas del día, con chicos y chicas de 18, 19, 20 años, las hormonas en efervescencia... puede ser complicado... (Entidad 7).

Si bien las entidades que disponen de pisos mixtos tienen buenas experiencias al respecto.

Es mucho mejor. $Y$ te diré una cosa, la convivencia funciona mucho mejor en el piso cuando son 2 y 2 que cuando hay 4 chicos [...] es que es más natural. (Entidad 11)

La mayor parte de los jóvenes tienen entre los 18 (39.8\%) y 19 años (31.9\%). Aunque también hay jóvenes de 20 años (18.7\%) y de 21 años (6\%) (media $=18$ años y 11 meses).

Respecto a la procedencia geográfica de los extutelados, la mitad de los jóvenes son de origen inmigrante $(54 \%)$, cosa que no se corresponde con la población tutelada. Esto se explica porque la mayor parte de estos jóvenes no tiene una familia en territorio español a la que acudir.

El 90\% de los jóvenes que en ese momento residían en los recursos no tenía trabajo, sólo un $8 \%$ tenía un contrato laboral, y un $2 \%$ trabajaba sin contrato. Como se comentaba anteriormente, los jóvenes inmigrantes tienen especiales dificultades para poder trabajar porque muchos no disponen del permiso de trabajo y tienen una escasa formación. 
Estamos hablando de que la población concretamente que hay ahora es toda inmigrante y sí que tienen el permiso de residencia, pero el de trabajo les es súper difícil conseguirlo. Un poco es el pez que se muerde la cola: "hasta que no tengas contrato, no te damos el permiso de trabajo" pero al revés, una empresa dice: "si no tienes permiso de trabajo..." tampoco están bien informados sobre el coste que les puede suponer, piensan que tienen que pagar mucho más y los chicos tampoco tienen una formación que tú digas: "ostras! Un empresario va a dar mucho la cara por este chaval" porque tienen una formación muy básica. [...] Entonces no paran de formarse, un curso tras otro, pero apenas les llega la oportunidad. (Entidad 3).

Los jóvenes que trabajan lo hacen, principalmente, en entidades protegidas que se dedican a la inserción de colectivos con dificultades. Estas entidades ofrecen la oportunidad de regularizar la situación laboral de los jóvenes, pero ofrecen contratos de formación con una continuidad limitada y sueldos que no permiten una autonomía económica.

Estas dificultades sumadas a la falta de oportunidades laborales explican que la mayor parte de jóvenes estén realizando alguna formación.
Ha habido un cambio importante. El perfil de hace 7 u 8 años era un perfil de joven que estaba trabajando y que todo esto venía mucho más cerrado. Aquí habría 2 elementos que se suman: por un lado, la ampliación de posibilidades formativas para los jóvenes como por ejemplo una prestación o como pueden ser las becas de "La Caixa" y por otro lado la situación de la crisis. (Entidad 14).

Respecto a la formación se observa una dicotomía entre los jóvenes que tienen como objetivo prioritario un proyecto formativo y los jóvenes que preferirían trabajar, pero a falta de oportunidades deciden estudiar. Así por un lado encontramos a un grupo que está estudiando un Ciclo Formativo de Grado Medio (30\%) o superior (3\%) o estudios universitarios (5\%). Por otro lado, encontramos a jóvenes que están realizando un Programa de Cualificación Profesional Inicial (28\%); cursos de alfabetización o lengua (15\%), Formación Ocupacional (7\%), ESO (8\%) o Acceso al grado Medio (2\%).

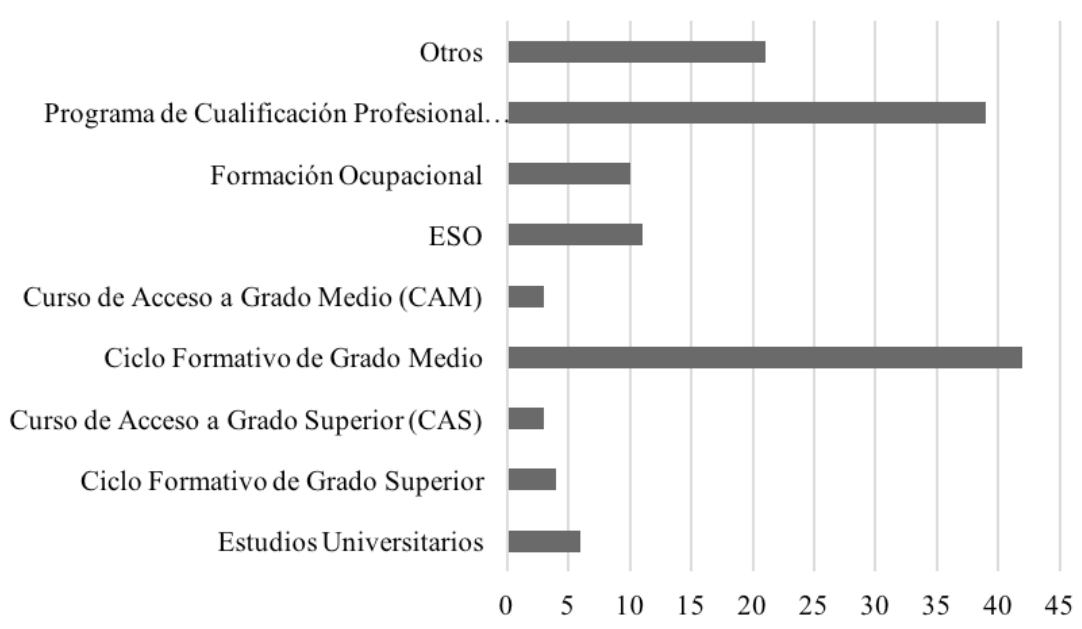

Gráfico 2. Estudios que realizan los jóvenes

Número de jóvenes según los estudios que cursan.

\subsection{Estancia y salida de los recursos residenciales}

El mayor abandono de los recursos se produce a los 20 años, siendo la permanencia promedio de 1 año y 8 meses. Las entidades coinciden en que 10 años atrás, se independizaban más temprano y que los jóvenes han ido alargando su estancia en los recursos residenciales debido a las dificultades de encontrar un trabajo.

Sin embargo, los factores que inciden en que el joven abandone el recurso antes de los 21 años son diversos, y no siempre se producen porque se han logrado los objetivos de autonomía propuestos.

Algunos jóvenes no tienen un proyecto claro o no encuentran sentido a su estancia en el recurso y acaban abandonándolo, ya sea por decisión propia o por decisión educativa. Otros deciden abandonar el recurso en cuanto consiguen una prestación, en muchos casos empujados por el deseo de no tener que cumplir con unas normas impuestas y dar explicaciones al educador. En el caso de las chicas, los educadores apuntan que abandonan 
el proyecto principalmente por las relaciones de pareja; si bien también tienen una actitud más decidida y autónoma que les permite emanciparse antes que a los chicos.

En las chicas les influye mucho el tema de pareja. Muchas se han ido a vivir con la pareja y tú te ponías las manos en la cabeza con un sentimiento paternalista brutal porque te hablaban de que querían ser madres con 19 o con 20 años y que "me quiero casar con este" y "quiero ser feliz con este". [...] Con pareja ha sido "bueno, me voy a vivir con tal", "me voy a vivir con cual" y "me voy a vivir con el otro". Bueno, también lo entiendes porque es lo que toca, pero claro, "abandonas todo un proceso de estudio tú que puedes llegar a la universidad si te da la gana y estás trabajando y todo tiene más o menos una estabilidad y una coherencia ¿quieres dejarlo todo por irte a vivir con esta persona?" - "Si'." (Entidad 21)

Por otro lado, hay jóvenes a los que les cuesta abandonar el centro. Algunos entrevistados atribuyen estos casos al vínculo emocional con el educador o con la entidad.

Sin embargo, las chicas que alargan su estancia suelen hacerlo para cumplir sus itinerarios formativos o por el hecho de ser madres, ya que cuentan con una mayor responsabilidad que les hace plantear este salto.

En algunos casos son los profesionales quienes deciden que el joven debe abandonar el recurso, de forma más o menos pactada, o bien por considerar que este ha logrado los objetivos por los cuales estaba en el piso o bien por situaciones límite en las que considera que el joven no puede continuar viviendo en el recurso. Las expulsiones están condicionadas principalmente por la falta de predisposición y el incumplimiento reiterado de la normativa del recurso mediante faltas leves - mal comportamiento, distorsión en la convivencia con los compañeros- o faltas graves, las cuales son consideradas como expulsiones directas, como, por ejemplo, agresiones, robos en el recurso o cometer algún delito. No obstante, algunos profesionales se cuestionan la aplicación estricta de las normas porque no serían tan estrictos con un hijo adolescente.

[...] la tercera vez que se llama la atención porque han encontrado a gente en el piso, porque hemos encontrado bebidas alcohólicas o porque no se va a la escuela, se le echa a la calle. Con eso quizá si somos demasiado estrictos y quizá por eso tenemos el piso vacío, no lo sé. Pero te lo cuestionas, ¿̇eh? Porque yo tengo hijos y a mis hijos les perdono muchas más cosas en la vida. En este sentido te lo digo. Claro que son mis hijos y estos no, pero... entiendes, ¿̇no? (Entidad 11)
Cuando la decisión de abandonar el recurso es tomada por el joven, suele estar motivada por la disconformidad con el control de los educadores, el cansancio de estar en el sistema de protección, porque el recurso no cumple sus expectativas o bien, en menor grado, porque aparece la posibilidad del retorno con la familia o de ir a vivir en pareja.

Los entrevistados señalan que cuando los jóvenes salen del centro la mayoría se va a vivir en pisos compartidos o alquila habitaciones. Una salida también frecuente para los jóvenes autóctonos es el retorno con la familia y la salida con la pareja o con la familia de la pareja, principalmente en las chicas. En el caso de la población inmigrante, el regreso a su país de origen no es una opción que contemplen por percibirla como un fracaso en su proceso migratorio.

La mayor parte de las entidades manifiesta que los jóvenes marchan sin trabajo o a lo sumo, con trabajos esporádicos o con malas condiciones laborales. La mayor parte sale-de los recursos habiendo estudiado CFGM ○ PCPI, cobrando la prestación no contributiva de la RMI y habiendo cobrado, durante su estancia en el recurso, las ayudas ofrecidas por el ASJTET. Los entrevistados también señalan que la red social de estos jóvenes es bastante limitada en el momento de su salida.

Gran parte de las entidades ofrece mantener el contacto a los jóvenes cuando abandonan, para acompañarlos y asesorarlos en problemas puntuales que puedan tener en los trámites de documentación y otras gestiones.

\section{Limitaciones}

El diseño de la investigación incluía la participación de todas las entidades del territorio catalán que acogen jóvenes tutelados $(n \circ=29)$, pero por motivos de disponibilidad de algunas entidades sólo pudimos acceder a 25 de ellas. En una de las entidades, la persona entrevistada no disponía de cargos de gestión como se requería. Esto fue debido a problemas de última hora en la agenda de la persona responsable del recurso. La substitución limitó las respuestas en aquellas preguntas más relacionadas con la gestión de los pisos y no se pudieron tener en cuenta en el análisis de los datos.

\section{Discusión}

Los datos del estudio nos han permitido conocer en mayor profundidad el programa de vivienda para apoyar el proceso de transición a la autonomía de los jóvenes extutelados, la labor de 
acompañamiento educativo, el perfil de los jóvenes y los profesionales, el acceso, la estancia y la salida de este programa. Sin lugar a duda, estos recursos cumplen con una función muy necesaria que puede ser determinante en los resultados de esta transición a la vida adulta. De hecho, como ya hemos señalado en el estudio Midwest (Courtney \& Dworsky, 2006; Courtney et al., 2007) se comprobó que los jóvenes que recibían estos apoyos tenían transiciones más exitosas. En este mismo sentido, Montserrat, Casas y Sisteró (2013), después de analizar el proceso de transición de los jóvenes atendidos por el ASJTET, concluyeron que los servicios de transición representan una mejora en los niveles educativos comparables con los jóvenes de la población general. El programa proporciona una necesaria estabilidad y guía en un período complejo como es la adolescencia en la que el joven, sin una red de apoyo como es la familia, puede seguir trayectorias vitales con riesgos difíciles de revertir.

Muchos de los chicos parecen aprovechar satisfactoriamente el recurso, aunque sorprende que en un entorno como el español con altas tasas de paro y graves dificultades para acceder a la vivienda el $70 \%$ de los jóvenes los abandonan antes de cumplir los 20 años. El informe presentado por el ASJTET (2011) apuntaba que el 59\% de los jóvenes tenía una salida que sus educadores valoraba como adecuada; pero el 29\% había conseguido los objetivos de forma parcial y un $11 \%$ no seguía el plan de trabajo. En el estudio presentado por Montserrat, Casas y Sisteró (2013), el $47.5 \%$ de los jóvenes conseguía sus objetivos, un $28.4 \%$ abandonaba el programa por propia voluntad y un $24.2 \%$ tenía-resultados poco favorables. Los entrevistados han apuntado a que, si bien una parte de los jóvenes abandona el recurso por haber logrado la autonomía necesaria, otra parte considerable lo hace por razones como el incumplimiento de las normas, el no aprovechamiento del recurso y la insatisfacción hacia la supervisión educativa que es percibida como controladora; aspecto que también se ha observado en otros países (Munson, Stanhope, Small \& Atterbury, 2017). Estos datos no dejan de ser preocupantes si además consideramos que muchos jóvenes no llegan a entrar en este tipo de recurso por falta de las habilidades de autonomía requeridas o por no tener un proyecto formativo claro, y requieren de un análisis en profundidad. Por otra parte, el estudio de Olson, Scherer, y Cohen (2017) señala que estos jóvenes tendrían menores habilidades en la toma de decisiones que sus pares no tutelados.

Una de las primeras preguntas que surgen al respecto es ¿Por qué muchos jóvenes acogidos en el sistema de protección no llegan a los 18 años con las habilidades mínimas básicas para poder iniciar una fase de aprendizaje en un piso compartido? En este sentido, es posible que los programas educativos para menores de edad se centren especialmente en cubrir las necesidades básicas y en el acceso a la escolaridad y los servicios de salud y no desplieguen suficientes esfuerzos en desarrollar las competencias básicas de autonomía que la mayoría de los jóvenes aprenden en su hogar (limpieza, compra, cocina, colada, gestión del dinero, gestionar las visitas de los amigos y responsabilizarse de su comportamiento durante las visitas, etc.). También debe tenerse en cuenta que muchos de los jóvenes que disfrutan del programa de vivienda fueron tutelados ya en la adolescencia e ingresan con unos hábitos y valores ya consolidados difíciles de cambiar en el escaso tiempo con el que cuentan los centros. De hecho, la mayoría de los participantes del programa son jóvenes inmigrantes no acompañados, una parte de los cuales, previamente a su viaje a España, ha vivido en la calle, en entornos hostiles y con valores necesarios para la supervivencia en la calle que pueden entrar en conflicto con las normas el programa.

Otro factor que considerar es la alta tasa de fracaso escolar en esta población (Montserrat, Casas y Baena, 2015). Cuando la experiencia escolar diaria conlleva a la convicción de que "no sirvo para estudiar" y no se ha tenido oportunidad de descubrir cuáles son las habilidades especiales que uno posee, es difícil poder visualizar un proyecto formativo en el que se pueda tener éxito. Este es un aspecto muy urgente que atender. Muchos de los niños tutelados han tenido déficits escolares importantes antes de la tutela. Los centros no deberían darse por satisfechos solo con asegurar que los niños acudan a la escuela a diario, deberían desarrollar proyectos educativos que sean capaces de dar apoyo individualizado para lograr reducir los déficits o atrasos que los niños puedan tener y apoyarles en su progresión, además de ayudarles a descubrir cuáles son sus talentos y vocaciones.

La vivencia del acompañamiento educativo como "control" es otro de los aspectos que frecuentemente se mencionan como causa de la salida de los jóvenes. A pesar de que en nuestro país contamos con educadores mejor formados para el acompañamiento educativo (Sala, Arnau, Courtney \& Dworsky, 2016), la falta de implicación de los jóvenes es un problema que también se da en otros países. Muchos de los entrevistados han manifestado sus dilemas respecto a la necesidad de hacer respetar las normas y la necesidad de una actitud paciente con los adolescentes. La relación educativa es un aspecto que requiere de un estudio en 
mayor profundidad. Sin embargo, esta orientación es fundamental cuando un alto porcentaje de jóvenes llegan a la mayoría de edad sin tener un plan claro (Lemus, Farruggia, Germo \& Chang, 2017), ni todos los jóvenes tutelados tienen perfiles similares ni las mismas necesidades (Miller, Paschall \& Azar, 2017). De hecho, Rome y Raskin (2017) hallaron que el acompañamiento de un adulto es un factor de protección en esta transición.

Por otra parte, uno de los motivos de abandono aludidos es la dificultad de tener que convivir con jóvenes que ellos no han escogido y por los que pueden no sentir afinidad. De hecho, el análisis del tipo de recursos del programa muestra que la mayor parte de recursos son pisos compartidos con supervisión discontinua que requieren que el joven disponga de un alto nivel de autonomía y responsabilidad, y son pocos los recursos para jóvenes con menos autonomía (supervisión continua). Uno de los recursos que ofrece el ASJTET que no se ha descrito aquí por no ser estrictamente un servicio residencial, es el SAEJ (Servicio de Acompañamiento Especializado para Jóvenes tutelados y extutelados). Este servicio ofrece acompañamiento educativo intensivo a jóvenes que viven en pisos u habitaciones de alquiler disponibles en el mercado. Este tipo de acompañamiento puede ser más normalizador, no obliga al joven a romper con su pequeña red de conocidos, amigos u otras personas de apoyo para irse a vivir al recurso que tiene una plaza disponible. También se podrían considerar otras alternativas para jóvenes que no pueden adaptarse a los recursos disponibles. En Illinois, en estos casos aplican unos "contratos alternativos" con un adulto de referencia que el joven escoge, siempre y cuando la Administración valore que es un entorno seguro y el adulto pueda proporcionar la supervisión necesaria (Sala, Arnau, Courtney \& Dworsky, 2016).

Las dificultades de acceso al mercado laboral han sido uno de los temas recurrentes en las entrevistas. En este sentido las entidades recurren a programas externos para jóvenes normalizados o para poblaciones en riesgo de exclusión. El ASJTET cuenta con un equipo de siete insertores laborales que ofrece orientación a los educadores y los jóvenes que lo solicitan, y convenia con algunas entidades estos programas externos, pero no hay programas específicos para jóvenes tutelados. Algunos países disponen de programas de inserción específicos porque consideran que los déficits competenciales para acceder al mercado laboral de los jóvenes tutelados son más graves que los de la mayoría de los jóvenes desfavorecidos que acuden a estos programas y que por ello no pueden aprovecharlos adecuadamente (Sala, Arnau, Courtney \& Dworsky, 2016).

No debemos olvidar que la mitad de los jóvenes del programa de vivienda son jóvenes inmigrantes no acompañados con permiso de residencia, pero sin permiso de trabajo, lo que supone una barrera de acceso a los programas de formación y al mercado de trabajo. Este es uno de los aspectos por el que expresaron mayor preocupación los entrevistados y que requeriría de la Administración el diseño de algún tipo de medida al respecto.

Finamente, en el estudio se apunta a dos perfiles de jóvenes: los que tienen un proyecto formativo claro a los que la estancia en los recursos les aporta la seguridad en aspectos como la economía o la vivienda para continuar con sus estudios, y por ello prolongan su estancia. Y, por otro lado, un grupo de jóvenes que tiene un proyecto de vida enfocado a la inserción laboral, que no encuentra trabajo o bien no dispone de autorización para trabajar y realiza formaciones poco cualificadas con la esperanza de encontrar unas prácticas en empresa que les dé una oportunidad laboral. En estos casos, la decepción por no poder cumplir las expectativas puede ser la causa del incumplimiento de los pactos realizados con el educador, del malestar y del abandono del recurso. Sería interesante explorar la opción de crear una red de empresas sensibilizadas con estos jóvenes que les ofreciera opciones reales de inserción.

Son necesarios más estudios que analicen en profundidad la relación educativa y la satisfacción con los recursos desde la óptica de los jóvenes atendidos.

\section{Listado de siglas utilizado}

ASJTET: Área de Apoyo a los Jóvenes Tutelados y Extutelados. De sus siglas en catalán de Àrea de Suport als Joves Tutelats i Extutelats.

CAM: Curso de Acceso a Ciclos Formativos de Grado Medio

CAS: Curso de Acceso a Ciclos Formativos de Grado Superior

CFGM: Ciclo Formativo de Grado Medio

CFGS: Ciclo Formativo de Grado Superior

CRAE: Centro Residencial de Acción Educativa

ESO: Educación Secundaria Obligatoria

FEPA: Federación de Entidades con Pisos y Proyectos Asistidos

PCPI: Programa de Cualificación Profesional Inicial

RMI: Renta Mínima de Inserción 


\section{Referencias bibliográficas}

Àrea de Suport als Joves Tutelats i Extutelats. (2011). Informe 2011. Retrieved from http://benestar.gencat.cat/web/. content/O3ambits_tematics/O7infanciaiadolescencia/temes_relacionats/jornades_treball_dgaia_2012/docs_2O_abril/ asjtet.pdf

Bravo, A., \& Santos-González, I. (2017). Menores extranjeros no acompañados en España: necesidades y modelos de intervención. Psychosocial Intervention, 26(1), 55-62.

Bravo, A., \& Fernández del Valle, J. (2001). Evaluación de la integración social en acogimiento residencial. Psichothema, 13(2), 197-204.

Bravo, A., \& Fernández del Valle, J. (2003). Las redes de apoyo social de los adolescentes acogidos en residencias de protección. Un análisis comparativo con población normativa. Psichothema, 15(1), 136-141.

Cameron, L., Hynes, A., Maycock, Y., O’Neill, E., \& O’Reilly, A. M. (2017). Promoting Resilience amongst Young People Transitioning from Care to Independent Living: Experiences of Residential Social Care Workers. Journal of Social Care, $7(1), 8$

Casas, F., Montserrat, C., \& Malo, S. (2010). Spanish national report summary: Education and young people from a public care background pathways to education in Spain. The YIPPEE project. Retrieved from http://tcru.ioe.ac.uk/ yippee

Clemens, E. V., Helm, H. M., Myers, K., Thomas, C., \& Tis, M. (2017). The voices of youth formerly in foster care: Perspectives on educational attainment gaps. Children and Youth Services Review.

Courtney, M. E. (2009). The Difficult Transition to Adulthood for Foster Youth in the US: Implications for the State as Corporate Parent. Social Policy Report, 23(1), 3-18.

Courtney, M. E., \& Hook, J. L. (2017). The potential educational benefits of extending foster care to young adults: Findings from a natural experiment. Children and youth services review, 72, 124-132.

Courtney, M. E., \& Okpych, N. J. (2017). Memo from CalYOUTH: Early findings on the relationship between extended foster care and youths' outcomes at age 19. Chicago, IL: Chapin Hall at the University of Chicago. Consultable en: http://www.chapinhall.org/sites/default/files/CY_EF_IBo317.pdf

Courtney, M. E., Dworsky, A. L., Cusick, G. R., Havlicek, J., Perez, A., \& Keller, T. E. (2007). Midwest evaluation of the adult functioning of former foster youth: Outcomes at age 21. Chicago: Chapin Hall Center for Children at the University of Chicago. Retrieved from http://www.chapinhall.org/sites/default/files/ChapinHallDocument_.pdf

Courtney, M. E., Dworsky, A., Keller, T., Havlicek, J., \& Bost, N. (2005). Midwest evaluation of the adult functioning of former foster youth: Outcomes at age 19. Chicago: Chapin Hall Center for Children at the University of Chicago. Retrieved from http://www.chapinhall.org/sites/default/files/ChapinHallDocument_4.pdf

Courtney, M. E., Hook, J. L., \& Lee J. S. (2010). Distinct Subgroups of Former Foster Youth during Young Adulthood: Implications for Policy and Practice. Chicago: Chapin Hall Center for Children at the University of Chicago.

Courtney, M. E., Piliavin, I., Grogan-Kaylor, A., \& Nesmith, A. (2001). Foster youth transitions to adulthood: A longitudinal view of youth leaving care. Child Welfare, $80(6):$ 685-717.

Courtney, M. E., Piliavin, I., Grogan-Kaylor, A., \& Nesmith, A. (1998). Foster youth transitions to adulthood: Outcomes 12 to 18 months after leaving out-of-home care. Institute for Research on Poverty, University of Wisconsin-Madison.

Courtney, M. E., \& Dworsky, A. (2006). Midwest evaluation of the adult functioning of former foster youth: Outcomes at age 19. ILLINOIS. Chicago: Chapin Hall Center for Children at the University of Chicago. Retrieved from http:// www.chapinhall.org/sites/default/files/ChapinHallDocument_5.pdf.

Dworsky, A., \& Gitlow, E. (2017). Employment outcomes of young parents who age out of foster care. Children and Youth Services Review, 72, 133-140.

Fernández del Valle, J., Sainero, A. M., \& Bravo, A. (2011). Salud mental de menores en acogimiento residencial. Badajoz: Servicio Extremeño de salud.

Festinger, T. (1983). No one ever asked us...A postscript to foster care. New York: Columbia University Press.

Forsman, H., Brännström, L., Vinnerljung, B., \& Hjern, A. (2016). Does poor school performance cause later psychosocial problems among children in foster care? Evidence from national longitudinal registry data. Child abuse \& neglect, 57, 61-71.

Fowler, P. J., Marcal, K. E., Zhang, J., Day, O., \& Landsverk, J. (2017). Homelessness and aging out of foster care: A national comparison of child welfare-involved adolescents. Children and Youth Services Review, 77, 27-33.

Geiger, J. M., \& Beltran, S. J. (2017). Experiences and outcomes of foster care alumni in postsecondary education: A review of the literature. Children and Youth Services Review, 79(C), 186-197.

Generalitat de Catalunya. (2015). Mapa de serveis socials de Catalunya. Actualització de dades bàsiques. 2014. Infància, adolescència i joventut. Departament de Treball, Afers Socials i Famílies. Retrieved from http://treballiaferssocials.gencat.cat/web/.content/O3ambits_tematics/15serveissocials/estadistiques/mapa_serveis_socials/2014bis/ Infancia_adolescesncia_joventut_2014.pdf 
Gypen, L., Vanderfaeillie, J., De Maeyer, S., Belenger, L., \& Van Holen, F. (2017). Outcomes of children who grew up in foster care: Systematic-review. Children and Youth Services Review, 76, 74-83.

Heerde, J. A., Hemphill, S. A., \& Scholes-Balog, K. E. (2016). The impact of transitional programmes on post-transition outcomes for youth leaving out-of-home care: a meta-analysis. Health \& social care in the community.

Hook, J. L. \& Courtney, M. E. (2011). Employment outcomes of former foster youth as young adults: The importance of human, personal, and social capital. Children and Youth Services Review, 33(10): 1855-1865.

IARS (2009). Factors que incideixen en la inclusió sociolaboral després del desinternament. Informe de recerca. Retrieved from http://grupsderecerca.uab.cat/iars/sites/grupsderecerca.uab.cat.iars/files/Informe\%20final\%2Oestudi\%20èxit.pdf

Lemus, D., Farruggia, S. P., Germo, G., \& Chang, E. S. (2017). The plans, goals, and concerns of pre-emancipated youth in foster care. Children and Youth Services Review, 78, 48-55.

Lopez, M., Santos, I., Bravo, A., \& Fernández del Valle, J. (2013). El proceso de transición a la vida adulta de jóvenes acogidos en el sistema de protección infantil. Revisión de la investigación y respuestas. Anales de psicología, 29(1),187-196.

Martin, E. (2011). Apoyo social percibido en niños y adolescentes en acogimiento residencial. International Journal of Psychology and Psychological Therapy, 11(1), 107-120.

Martin, E., Muñoz, M. C., Rodríguez, T., \& Pérez, Y. (2008). De la residencia a la escuela: la integración social de los menores en acogimiento residencial con el grupo de iguales en el contexto escolar. Psicothema, 20(3), 376-382.

Martín, G., García, D., \& Siverio, M.A. (2012). Inadaptación autopercibida de los menores en acogimiento residencial. Anales de Psicología, 28 (2), 541-547.

Melendro, M. (Dir.). (2010). El tránsito a la vida adulta de los jóvenes en dificultad social. Madrid, UNED.

Melendro, M., \& Rodríguez, A.E. (2015). Los estudios sobre el tránsito a la vida adulta de jóvenes vulnerables y estrategias para su inclusión social. En Los estudios sobre la juventud en España: Pasado, presente, futuro. Revista de estudios de juventud. 110, 201-215.

Miller, E. A., Paschall, K. W., \& Azar, S. T. (2017). Latent classes of older foster youth: Prospective associations with outcomes and exits from the foster care system during the transition to adulthood. Children and Youth Services Review, 79, 495-505.

Montserrat, C., Casas, F., \& Bertran, I. (2013). Desigualdad de oportunidades educativas entre los adolescentes en acogimiento residencial y familiar. Infancia y Aprendizaje, 36.

Montserrat, C., Casas, F., \& Malo, S (2013). Delayed educational pathways and risk of social exclusion: The case of young people from public care in Spain. European Journal of Social Work, 16,1, 6-21.

Montserrat, C., Casas, F., González, M., Malo, S., Araujo, L., \& Navarro, D. (2010). Factors d'èxit en infants atesos en CRAE. Girona: Fundació Plataforma Educativa.

Montserrat, C., Casas, F., Malo, S., \& Bertran, I. (2011). Los itinerarios educativos de los jóvenes ex-tutelados. Madrid: Ministerio de Sanidad, Política Social e lgualdad.

Montserrat, C., Casas, F., \& Sisteró, C. (2013). Estudi sobre l'atenció als joves extutelats: Evolució, valoració i reptes de futur. Informe de resultats. Retrieved from http://www.fepa18.org/wp-content/uploads/2014/o1/Informe-Recerca-FEPA-Universitat-de-Girona-Juliol-2013.-DEFINITIU.pdf

Montserrat, C.; Casas, F., \& Baena, M. (2015). La educación de niños, niñas y adolescentes en el sistema de protección. Girona: Documenta Universitaria.

Munson, M. R., Stanhope, V., Small, L., \& Atterbury, K. (2017). "At times I kinda felt I was in an institution": Supportive housing for transition age youth and young adults. Children and Youth Services Review, 73, 430-436.

Observatorio de la Infancia (2016). Boletín de datos estadísticos de medidas de protección a la infancia. Retrieved from http://www.observatoriodelainfancia.msssi.gob.es/productos/pdf/Estadistica_basica_de_proteccion_a_la_infancia_17. $\mathrm{pdf}$

Olson, A., Scherer, D. G., \& Cohen, A. L. (2017). Decision-making skills of emerging adults aging out of foster care. Children and Youth Services Review.

París, M. E. C., Hernando, G. C., \& Martínez, R. M. G. (2017). El tránsito a la vida adulta de los jóvenes en acogimiento residencial: el rol de la familia. Educación $X X_{1}$.

Rome, S. H., \& Raskin, M. (2017). Transitioning Out of Foster Care: The First 12 Months. Youth \& Society, 0044118X17694968.

Sala-Roca, J., Arnau, L., Courtney, M. E., \& Dworsky, A. (2016). Programs and Services to help Foster Care Leavers during their Transition to Adulthood: A Study Comparing Chicago (Illinois) to Barcelona (Catalonia). Retrieved from https://ddd.uab.cat/pub/estudis/2016/158016/2016-05-26_summary_report.pdf

Sala-Roca, J., Villalba, A., Jariot, M., \& Arnau, L. (2012). Socialization process and social suport networks of out-of-care youngsters. Children and Youth Services Review, 34, 1015-1023. 


\section{CÓMO CITAR ESTE ARTÍCULO}

Comasòlivas, A., Sala-Roca, J., \& Marzo, T.E. (2018). Los recursos residenciales para la transición hacia la vida adulta de los jóvenes tutelados en Cataluña. Pedagogía Social. Revista Interuniversitaria, 31, 125-137. DOI: 10.7179/PSRI_2018.31.10

\section{DIRECCIÓN COMPLETA DE LOS AUTORES}

Antonio Comasòlivas Moya. Correo electrónico: toni.comasolivas@eveho.eu Dirección postal: Fundació Eveho. C/Regàs, 99. 08203 Sabadell

Josefina Sala-Roca. Correo electrónico: Fina.Sala@uab.cat Dirección postal: Facultat de Ciències de l'Educació, despacho G6-171 Universitat Autònoma de Barcelona. 08193 Cerdanyola del Vallès

Teresa Eulàlia Marzo Arpón. Correo electrónico: mmarzo@peretarres.org Correo postal: Facultad de Educación social y Trabajo social Pere Tarrés Santaló 37 08021- Barcelona

\section{PERFIL ACADÉMICO}

Antonio Comasòlivas Moya. Profesional de la atención a la infancia y adolescencia en riesgo en Fundació Eveho. Director de recurso residencial para jóvenes extutelados, director del Programa T-Jove y técnico del Área Tecnico-Pedagógica de dicha fundación. Doctorando en ciencias de la educación en la Universidad Autónoma de Barcelona (UAB). Investigador en formación del grupo de investigación de la UAB IARS (Infancia y Adolescencia en Riesgo Social). El ámbito de investigación desarrollado es: la transición a la vida adulta y los recursos de vivienda de los jóvenes tutelados y extutelados.

Josefina Sala-Roca. Profesora del departamento de Pedagogía Sistemática y Social de la Universidad Autónoma de Barcelona. Coordina el grupo de Investigación de Infancia y Adolescencia en Riesgo Social (IARS). El grupo IARS en colaboración con FEPA, FEDAIA y DGAIA ha desarrollado diversos estudios sobre los factores que inciden en el bienestar y desarrollo positivo de los jóvenes tutelados, así como de las dificultades y apoyos a su transición a la vida independiente. Las investigaciones desarrolladas se han publicado en diversos artículos e informes.

Teresa Eulàlia Marzo Arpón. Profesora de la Facultad de Educación social y Trabajo social Pere Tarrés-Universidad Ramon Llull (Barcelona). Es investigadora en el Grupo de Innovación y Análisis Social (GIAS) de la Universidad Ramon Llull y en el grupo de investigación de Infancia y adolescencia en riesgo social (IARS) de la Universidad Autónoma de Barcelona. Centra su actividad investigadora en la incidencia que ejerce el tiempo libre educativo en la infancia y la adolescencia, y en los factores que contribuyen al éxito en la mayoría de edad de la infancia y adolescencia tutelada. 
